\title{
Staphylococcus auricularis
}

National Cancer Institute

\section{Source}

National Cancer Institute. Staphylococcus auricularis. NCI Thesaurus. Code C86751.

A species of facultatively anaerobic, Gram positive, cocci shaped bacteria in the phylum Firmicutes. This species is catalase positive, urease, coagulase and oxidase negative and produces acid from fructose and glycerol. S. auricularis is a commensal organism of the human ear and may be pathogenic in immunocompromised individuals. 